\title{
The role of mesoscale eddies time and length scales on phytoplankton production
}

\author{
V. Pérez-Muñuzuri ${ }^{1}$ and F. Huhn ${ }^{2}$ \\ ${ }^{1}$ MeteoGalicia, Consellería de Medio Ambiente, Rúa Roma, 6, 15707 Santiago de Compostela, Spain \\ ${ }^{2}$ Group of Nonlinear Physics, Faculty of Physics, Univ. of Santiago de Compostela, 15781 Santiago de Compostela, Spain
}

Received: 30 October 2009 - Revised: 21 January 2010 - Accepted: 31 January 2010 - Published: 31 March 2010

\begin{abstract}
Horizontal mixing has been found to play a crucial role in the development of spatial plankton structures in the ocean. We study the influence of time and length scales of two different horizontal two-dimensional (2-D) flows on the growth of a single phytoplankton patch. To that end, we use a coupled model consisting of a standard three component ecological NPZ model and a flow model able to mimic the mesoscale structures observed in the ocean. Two hydrodynamic flow models are used: a flow based on Gaussian correlated noise, for which the Eulerian length and time scales can be easily controlled, and a multiscale velocity field derived from altimetry data in the North Atlantic ocean. We find the optimal time and length scales for the Gaussian flow model favouring the plankton spread. These results are used for an analysis of a more realistic altimetry flow. We discuss the findings in terms of the time scale of the NPZ model, the qualitative interaction of the flow with the reaction front and a Finite-Time Lyapunov Exponent analysis.
\end{abstract}

\section{Introduction}

Spatial heterogeneity or "patchiness" in phytoplankton distributions is an old oceanographic observation that dates back to the 12th century (Griffiths, 1939; Bainbridge, 1957) and is still a field of current research. Phytoplankton forms the base of the food chain and is responsible for a large amount of the biological primary production in the oceans. Therefore, it plays an important role for the entire marine ecosystem. Furthermore, it acts on the ocean's $\mathrm{CO}_{2}$ uptake, as a part of the carbon absorbed in phytoplankton by photosynthesis is transported to deep water, when dead plant material sinks

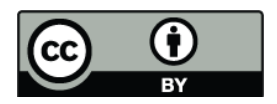

Correspondence to: V. Pérez-Muñuzuri (vicente.perez@cesga.es) down. A striking feature associated with the dynamics of phytoplankton populations is the occurrence of rapid and extensive bloom formations. Such events are characterised by a sharp rise in algae concentration of up to several orders of magnitude (Beltrami and Carroll, 1994) followed by a sudden collapse, whereby the phytoplankton populations return to their original low level. Some phytoplankton species are toxic, so their appearance in large numbers have the potential to damage higher organisms such as zooplankton, shellfish and fish. Given the impact of phytoplankton blooms, it is of interest to understand the dynamics of plankton growth and the conditions for a rapid and wide-spreading plankton patch.

A plankton population in the ocean can be seen as a biological system with predator-prey dynamics in a mobile environment that alters its spatial distribution. As the well-lit surface layer of the ocean is normally nutrient poor, a nutrient source is necessary to start a rise in phytoplankton concentration above a low stationary level. Apart from occasional aeolian dust deposition (Pasquero et al., 2005), upwelling of nutrient-rich water from deeper water layers or vertical mixing is assumed to be the main nutrient source (Mahadevan and Archer, 2000; Mahadevan and Tandon, 2006; Lévy, 2008; Oschlies and Garcon, 1998). A variety of hydrodynamical effects leading to vertical transport is reported (Lévy, 2008; Martin et al., 2001), many of which may be highly localized in space. They represent an important cause for plankton patchiness. Pasquero et al. (2005) showed that not only does the mean value of the nutrient flux influence the primary production but especially its temporal and spatial variability. The dependence of the primary production on temporal variability was analysed considering the intrinsic time scales of the plankton system. Martin et al. (2002) and Pasquero et al. (2005) found a lower primary production for nutrient sources correlated with eddy cores than for uncorrelated source positions.

Published by Copernicus Publications on behalf of the European Geosciences Union and the American Geophysical Union. 
Assuming the presence of a nutrient source, horizontal transport, due to the turbulent velocity field on the mesoscale and sub-mesoscale $(\sim 1-500 \mathrm{~km})$, modifies the patterns in the plankton distribution and influences the temporal development of plankton patches (Abraham, 1998; Martin, 2003; Lévy and Klein, 2004; McKiver et al, 2009). Lehahn et al. (2007) found that the gradients in chlorophyll concentration fields from satellite images align with unstable manifolds of the geostrophic velocity field which suggests that horizontal transport controls the chlorophyll patterns. See (Haller and Yuan, 2000; Wiggins, 2005) for a description of stretching and folding of a passive tracer in 2-D turbulence and an explanation of the concepts of a Lagrangian flow analysis that can also be applied to reactive tracers.

As the ocean is a multiscale system, a single plankton patch is advected and mixed by flow structures of very different time and length scales. The interaction of these scales with the parameters of the plankton system affects the spatiotemporal development of a plankton patch. A minimum width for phytoplankton filaments can be calculated, which is determined by a few parameters describing the flow and the growth of the plankton system (Martin, 2000; McLeod et al., 2002). Sandulescu et al. (2007) reported simulated localized plankton blooms in vortices in the wake of an island, when the long residence time of water masses in the vortices is comparable to the plankton growth time.

In this paper, we focus on the interplay of length and time scales of a 2-D horizontal flow with the plankton dynamical system. We investigate the response of a plankton model to a well-defined hydrodynamical forcing and address the question under which flow conditions does a plankton patch evolving from a single localized nutrient source spread optimally. We, therefore, chose a simple model flow defined by autocorrelation length and time and analyse the final size of the plankton patch. The results are compared to a similar analysis of plankton patches in a multiscale altimetry flow, including an example of two plankton patches where the effect of different length and time scales can be seen. We do not correlate nutrient upwelling regions to flow structures, as we investigate the response of the plankton system to basic spatiotemporal properties of a model flow.

The paper is organized as follows: Section 2 describes the numerical models for the hydrodynamic flow and the plankton system, as well as their coupling. In Sect. 3, the effect of flow time and length scales on plankton patch size is analysed. The final section comprises of the conclusions.

\section{Models}

The role of the mesoscale flow structures for the evolution of a phytoplankton patch is studied considering a 2-D incompressible flow, so that the velocity field is given by $\boldsymbol{V}=\boldsymbol{e}_{z} \times \nabla \psi$, where $\psi(x, y, t)$ is a time-dependent stream function. An ecological model coupled to the flow models the phytoplankton production.

\subsection{The hydrodynamic models}

To study the effects of length and time scales of a flow on a phytoplankton patch, we use two flow models, an analytic one and another more realistic one. Figure 1 shows the two stream functions corresponding to the two depicted flows. An analytic Gaussian correlated flow is especially appropriate in investigating the influence of time and length scales on plankton production, because its time and spatial scales are independent and can be adjusted a priori. The obtained results for this artificial flow are compared to a similar analysis for a flow derived from satellite altimetry measurements.

\subsubsection{Gaussian correlated flow}

The Gaussian correlated flow is defined in terms of a stream function with dynamics represented by a Gaussian spatiotemporal distributed noise. The noise has zero mean and the spatiotemporal autocorrelation function $G(\rho, s)$ is given by

$$
\begin{aligned}
\left\langle\psi(\boldsymbol{r}, t) \psi\left(\boldsymbol{r}^{\prime}, t^{\prime}\right)\right\rangle= & G\left(\left|\boldsymbol{r}-\boldsymbol{r}^{\prime}\right|,\left|t-t^{\prime}\right|\right)= \\
& \Upsilon\left(\left|\boldsymbol{r}-\boldsymbol{r}^{\prime}\right| / \lambda\right) \Theta\left(\left|t-t^{\prime}\right| / \tau\right),
\end{aligned}
$$

with $\boldsymbol{r}=(x, y)$. The temporal correlation function, $\Theta(s / \tau)$, describes an Ornstein-Uhlenbeck (OU) process given by

$\Theta(s / \tau)=\frac{1}{\tau} \exp (-s / \tau)$

where $\tau$ corresponds to the correlation time for the OU process and $s=\left|t-t^{\prime}\right|$. The spatial correlation is

$\Upsilon(\rho / \lambda)=\frac{\sigma^{2}}{2 \pi \lambda^{2}} \exp \left(-\rho^{2} / 2 \lambda^{2}\right)$,

where $\sigma^{2}$ is the noise intensity, $\lambda$ is the correlation length and $\rho=\left|\boldsymbol{r}-\boldsymbol{r}^{\prime}\right|$. In order to study the effects of noise, the noise dispersion $G(0,0)$ is kept constant

$$
G(0,0)=\frac{\sigma^{2}}{2 \pi \tau \lambda^{2}}=\text { const }
$$

while varying $\tau$ or $\lambda$. Thus, an artificial flow is defined by specific, typical Eulerian time and length scales for the velocity. Details on the numerical generation of a spatiotemporal correlated noise given by Eqs. (2-4) are given in Alonso et al. (2002) and Sagués et al. (2007).

\subsubsection{Altimetry flow}

For the more realistic flow case, we consider surface currents derived from satellite altimetry data provided by AVISO. The Ssalto/Duacs system processes data from all altimeter missions (Jason-1\&2, TOPEX/Poseidon, Envisat, GFO, ERS$1 \& 2$ and Geosat) and merges the data from all available 
(a)

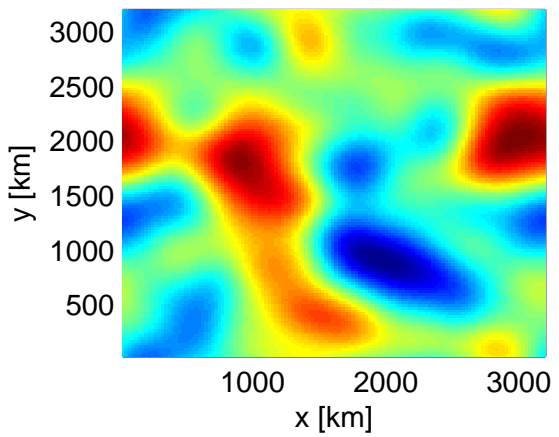

(b)

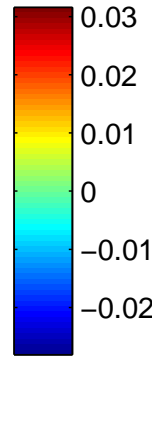

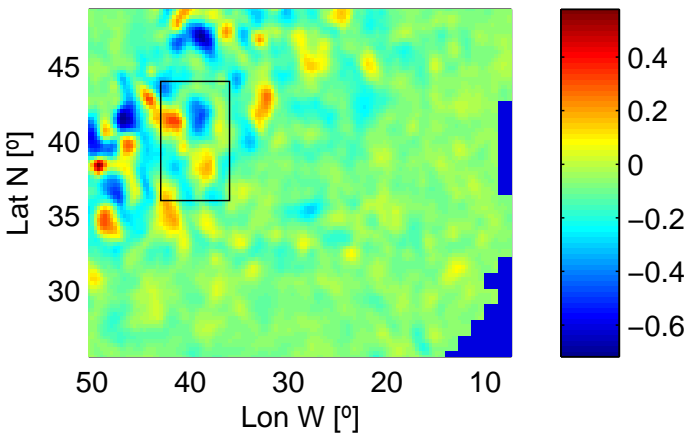

Fig. 1. Stream function for the Gaussian model (a) and the altimetry data (b). Parameters: (a) $\tau / T=1, \lambda / L=10$, and $N_{x}=N_{y}=128$; (b) the area of simulation spans from $25^{\circ} \mathrm{N}$ to $49^{\circ} \mathrm{N}$, and from $7^{\circ} \mathrm{W}$ to $50^{\circ} \mathrm{W}\left(N_{x}=130, N_{y}=90\right)$. The marked area in the altimetry data is analysed in detail later. The visible land structure is the coast of Spain and Morocco.

satellites. The global maps of Sea Level Anomaly (SLA) are about a seven-year mean and have a spatial resolution of $(1 / 3)^{\circ}$ on a Mercator grid and a temporal resolution of 1 day. For this dataset, geostrophic velocities are estimated as

$V_{x}=-\frac{g}{f R_{T}} \frac{\partial \psi_{\mathrm{SLA}}}{\partial \phi} \quad V_{y}=\frac{g}{f R_{T} \cos \phi} \frac{\partial \psi_{\mathrm{SLA}}}{\partial \ell}$

where $\psi_{\text {SLA }}$ is the SLA, $g$ is the gravity, $f$ the Coriolis parameter, $R_{T}$ the Earth radius, $\phi$ the latitude and $\ell$ the longitude, and the derivatives were calculated with finite differences. The analysed data is from 12 November 2008. We chose the area to include a wide range of velocity absolute values and different local length scales, always keeping in mind that the geostrophic velocities from altimetry data are only a rough estimate of the ocean currents and altimetry data only resolves mesoscale structures.

\subsection{NPZ model}

We use a well-established NPZ ocean ecosystem model representing the plankton dynamics (Fasham et al., 1990; Oschlies and Garcon, 1999; Franks, 2002; Martin et al., 2002; Pasquero et al., 2005). It is a three component model describing the interaction of three species of the trophic chain: nutrients $N$, phytoplankton $P$, and zooplankton $Z$. Their concentrations evolve according to the following equations (Pasquero et al., 2005; Sandulescu et al., 2007, 2008),

$$
\begin{aligned}
\dot{N}=F_{N}= & \Phi_{N}-f(N, P)+ \\
& +\mu_{N}\left((1-\gamma) g(P, Z)+\mu_{P} P+\mu_{Z} Z^{2}\right) \\
\dot{P}=F_{P}= & f(N, P)-g(P, Z)-\mu_{P} P \\
\dot{Z}=F_{Z}= & \gamma g(P, Z)-\mu_{Z} Z^{2}
\end{aligned}
$$

and

$f(N, P)=\beta \frac{N}{k_{N}+N} P$

$$
g(P, Z)=\frac{\alpha \eta P^{2}}{\alpha+\eta P^{2}} Z .
$$

The reader is referred to (Oschlies and Garcon, 1999; Pasquero et al., 2005) for a detailed description of the model including an explanation of the different interaction terms.

Since we assume a 2-D flow, vertical mixing, which is a determinant factor for phytoplankton formation, must be parameterized. We consider a constant high nutrient concentration $N_{0}$ below the mixed layer (Martin et al., 2002; Pasquero et al., 2005). The upward nutrient flux then is

$\Phi_{N}=S(x, y)\left(N_{0}-N\right)$,

where the function $S(x, y)$ determines the strength of the upwelling depending on the position in the flow. $1 / S(x, y)$ is the corresponding relaxation time. Equation (8) reflects the fact that nutrients are brought up to the surface from deep waters via turbulent mixing or upwelling. The parameters used are taken from (Pasquero et al., 2005; Sandulescu et al., 2007, 2008) and are shown in Table 1. For this set of parameters, the model dynamics exhibits stationary behaviour in the long-term limit. To obtain dimensionless values, all quantities and parameters in the model are measured in units of length $L=25 \mathrm{~km}$, time $T=30$ days and nitrogen mass $M=10^{12} \mathrm{mmol} \mathrm{N}$ following Sandulescu et al. (2007).

\subsection{Numerical methods and initial conditions}

The coupling of the hydrodynamic and NPZ models yields a reaction-diffusion-advection system described by the following set of partial differential equations:

$\frac{\partial \mathbf{C}}{\partial t}+(\mathbf{V} \cdot \nabla) \mathbf{C}=\mathbf{F}(\mathbf{C})+D \nabla^{2} \mathbf{C}$.

Here, $\mathbf{C}=[N, P, Z]$ and $\mathbf{F}=\left[F_{N}, F_{P}, F_{Z}\right]$ is given by Eqs. (6-8). For 2-D incompressible flows, the velocity $\mathbf{V}=$ $\left[V_{x}, V_{y}\right]$ of an advected particle is given by $\boldsymbol{V}=\boldsymbol{e}_{z} \times \nabla \psi$. 
Table 1. List of parameters used in the NPZ model, Eqs. (6-8).

\begin{tabular}{lll}
\hline parameter & value & $\begin{array}{l}\text { dimensionless } \\
\text { value }\end{array}$ \\
\hline$\beta$ & $0.66 \mathrm{~d}^{-1}$ & 19.8 \\
$\eta$ & $1\left(\mathrm{mmol} \mathrm{N} \mathrm{m}^{-3}\right)^{-2} \mathrm{~d}^{-1}$ & 0.12288 \\
$\gamma$ & 0.75 & 0.75 \\
$\alpha$ & $2 \mathrm{~d}^{-1}$ & 60 \\
$S_{l}$ & $0.00648 \mathrm{~d}^{-1}$ & 0.1944 \\
$S_{h}$ & $0.648 \mathrm{~d}^{-1}$ & 19.44 \\
$k_{N}$ & $0.5 \mathrm{mmol} \mathrm{N} \mathrm{m}^{-3}$ & 7.8125 \\
$\mu_{N}$ & 0.2 & 0.2 \\
$\mu_{P}$ & $0.03 \mathrm{~d}^{-1}$ & 0.9 \\
$\mu_{Z}$ & $0.2\left(\mathrm{mmol} \mathrm{N} \mathrm{m}^{-3}\right)^{-1} \mathrm{~d}^{-1}$ & 0.384 \\
$N_{0}$ & $8 \mathrm{mmol} \mathrm{N} \mathrm{m}^{-3}$ & 125 \\
\hline
\end{tabular}

The stream functions $\psi(x, y, t)$ are described in Sect. 2.1. Following Sandulescu et al. (2007), we use a constant value for the diffusion of all the biological fields $D=10 \mathrm{~m}^{2} / \mathrm{s}$ which is given by the Okubo estimation (Okubo, 1971). The dimensionless value used was $D=0.04147$. Nevertheless, for the scales considered here, diffusion of biological tracers plays a limited role and the dominant effect is the explicit spatial advection (see also Bracco, 2009). The reactiondiffusion-advection problem was integrated numerically on a $N_{x} \times N_{y}$ square lattice using a semi-Lagrangian algorithm (Staniforth and Cote, 1991; Spiegelman and Katz, 2006; Pasquero et al., 2005; Sandulescu et al., 2007) with spatial step size $\Delta x=1.0$ and time step $\Delta t=10^{-3}$. The algorithm induces a numerical diffusion that is smaller than the real diffusion for the biological tracers (Sandulescu et al., 2007) and the spatial advection.

As an initial condition, all biological concentrations were set to their steady value, $N_{0}=0.185, P_{0}=0.355$ and $Z_{0}=0.444 \mathrm{mmol} \mathrm{N} \mathrm{m}^{-3}$ for a low nutrient flux with $S=$ $S_{l}=0.00648 \mathrm{~d}^{-1}$, simulating a nutrient-poor domain $\left(P_{p_{0}}=\right.$ $0.0633 \mathrm{mmol} \mathrm{N} \mathrm{m}^{-3} \mathrm{~d}^{-1}$ ). In order to start the evolution of a plankton patch, we consider the effect of local upwelling and assume local, strong, vertical mixing leading to a nutrientrich spot in the mixed layer. This is numerically simulated by imposing a hundred-times larger value of the nutrient transfer rate $S=S_{h}=0.648 \mathrm{~d}^{-1}$ on a small region of $3 \times 3$ grid points $(75 \mathrm{~km} \times 75 \mathrm{~km})$. For each run, the stream function for the flow is random, so the position of the nutrient source, always in the middle of the domain, is uncorrelated with the flow. The upwelling is switched on for an active time $T_{f}$ after which $S$ is set to its low value again. In this paper, we keep constant the active period of $T_{f} / T=3$ ( $T_{f}=90$ days). The influence of a periodic active time $T_{f}$ on the primary production was analysed by Pasquero et al. (2005). Periodic boundary conditions were imposed on the concentrations and velocity gradients for the Gaussian flow model. To quan-

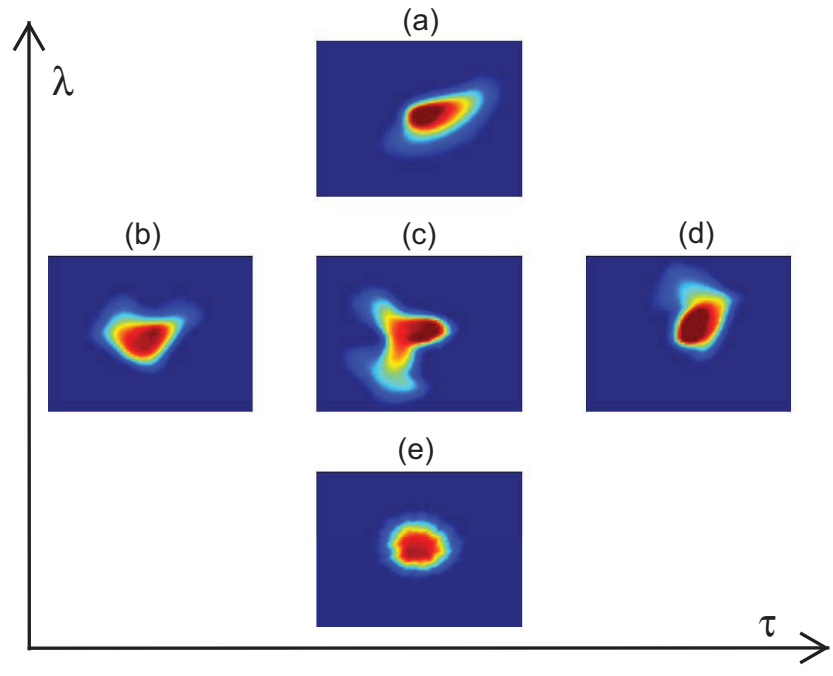

Fig. 2. Phytoplankton patches observed for different pairs of time and length scales $(\tau, \lambda)$ for the Gaussian flow, Eqs. (2-4). Length scale increases from bottom to top, and the temporal correlation scale from left to right. Note the larger patch size for intermediate values of $\tau$ and $\lambda$. Set of parameters: $G(0,0)=225$, $N_{x}=N_{y}=128$. Length and time scales $\left(\log _{10}(\tau / T), \log _{10}(\lambda / L)\right)$ : (a) $(-0.5,1.5)$, (b) $(-1.25,1)$, (c) $(-0.5,1)$, (d) $(1.5,1)$, (e) $(-0.5,-0.5)$

tify the phytoplankton population as a function of the time and length scales of the flow, we compute the plankton patch area $B_{s}$ as the area where the phytoplankton concentration $P$ is clearly larger than the steady state concentration $P_{0}$

$B_{S}=\int_{P>1.1 P_{0}} d A$

Maximal plankton concentrations in the patch are approximately $P / P_{0} \approx 1.5$, so the criterion of $P>1.1 P_{0}$ is adequate to separate the patch from the background concentration.

\section{Results}

\subsection{Gaussian flow - optimal time and length scales}

The effect of how time and length scales of the flow act on the development of a phytoplankton patch is shown in Fig. 2 for the Gaussian flow model. In this case, a nutrient source with a high transfer rate $S=S_{h}$ and a size of $3 \times 3$ grid points was set at the center of the $128 \times 128$ domain and initiated the development of a plankton patch. The advective flow is obtained from the Gaussian correlated stream function defined by length scale $\lambda$ and time scale $\tau$. During the whole simulation of 30 days the nutrient source was switched on. Note that for the intermediate values of the time and length scales (panel c) the advective velocity field interacts especially with the expanding reaction front which results in a 

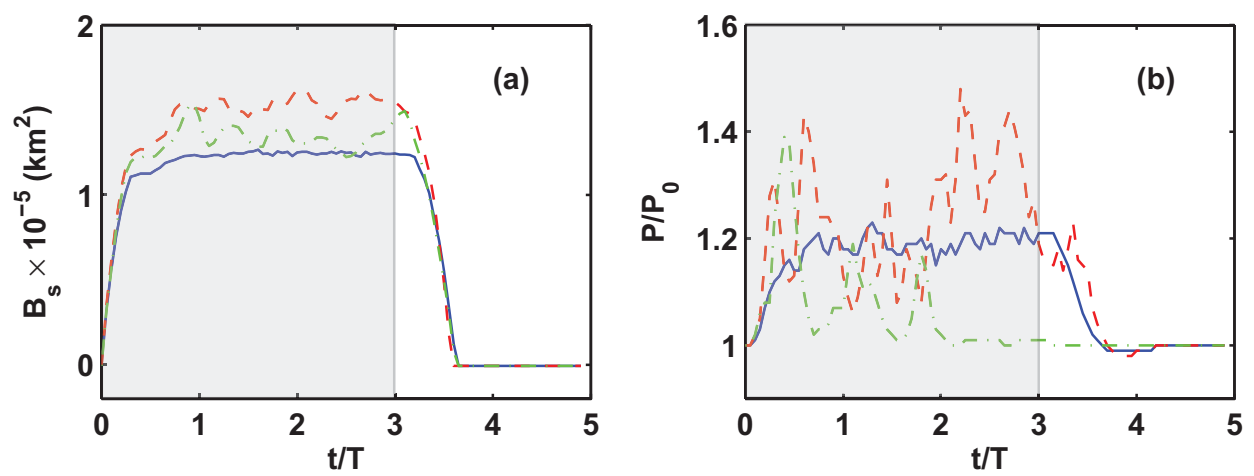

Fig. 3. Time evolution of the phytoplankton patch size $B_{S}(\mathbf{a})$ and the phytoplankton concentration 7 grid points apart from the upwelling region (b) for three pairs of Eulerian time and length scales $(\tau, \lambda)$ for the Gaussian flow, Eqs. $(2-4)$. Blue solid $\operatorname{line}\left(\log _{10}(\tau / T), \log _{10}(\lambda / L)\right)=$ $(-0.5,-0.5)$, red dashed line $(-0.5,1)$ and green dashed-dotted line $(-0.5,1.5)$. Set of parameters: $G(0,0)=225, N_{x}=N_{y}=128$ and active upwelling time $T_{f} / T=3$ (gray shade).

folded structure of the front and a larger patch size than in the other cases. In the vertical three images, with different length scales (panels a, c, e), it is apparent that for small length correlations (panel e) the patch grows mostly as a circular wave with small scale perturbations of the front, whereas for large length scales (panel a) the patch is just advected to the right. The horizontal three images with different time scales (panels b, c, d) shows that for intermediate values of the time correlation, the patch is most deformed and has areas of still low but increasing plankton concentrations (green). This behaviour might correspond to a resonant behaviour between the flow time scale and the time scale of the NPZ model, analysed in detail later.

To give a qualitative understanding, why optimal Eulerian time and length scales for the growth of the phytoplankton patch in the Gaussian flow can be expected, we consider the two limiting cases of $\tau \rightarrow 0$ and $\tau \rightarrow \infty$ for a constant $\lambda$. For $\tau \rightarrow 0$ and finite dispersion, Eq. (4), the intensity of the flow $\sigma^{2}$ tends to zero and the stream function vanishes. In this case, the diffusive non-advective case is recovered. For $\tau \rightarrow \infty$ a steady unidirectional flow is obtained. In this case, relevant fluctuations of the flow are infrequent and their effects on the system are small (Lorenzo et al., 2003; Sagués et al., 2007). The two limiting cases for $\lambda$ can be found with an analogous reasoning (Eq. 4). For $\lambda \rightarrow 0$ the totally uncorrelated diffusive case is reached and for $\lambda \rightarrow \infty$ the unidirectional flow cannot fold and distort the reaction front.

The temporal evolution of the phytoplankton patch size for different Eulerian time and length scales is shown in Fig. 3a. Note that independently of $\tau$ and $\lambda$, a steady patch size is rapidly attained after an initial time $T_{1} \approx 0.3 T \approx 9$ days. It decays to zero after the nutrient source is switched off for times $t / T>T_{f} / T$. The size of the plankton patch is largest for intermediate values of $\tau$ and $\lambda$ (red dashed line in panel a), as explained above. For an Eulerian flow time scale $\tau$, order of magnitude of the initial time $T_{1}$, the patch size is maximal which suggests a kind of resonant behaviour between the flow and the plankton dynamical system. At an exemplary grid point close to the nutrient source, initially, the plankton concentration rapidly increases due to a high nutrient flux which is caused by the short relaxation time $S_{h}^{-1}$ and the large nutrient difference $\left(N_{0}-N\right)$ (Fig. 3b). During the active period $T_{f}$, flow fluctuations give rise to changes in the phytoplankton concentration, when different fluid parcels are advected over the chosen grid point. Note that these fluctuations are larger and more frequent for intermediate values of $\tau$ and $\lambda$ corresponding to a distorted reaction front that curls and folds continuously. Finally, the plankton concentration relaxes towards the stationary value.

The obtained results for the Gaussian flow are summarized in Fig. 4 where the mean plankton patch size is represented as a function of Eulerian time and length scales of the flow. $\lambda$ and $\tau$ are both varied independently over more than two orders of magnitude $(2.5 \mathrm{~km}<\lambda<790 \mathrm{~km}$ and 0.3 days $<\tau<950$ days) exploring typical ocean mesoscales and beyond. For a fixed parameter pair $(\tau, \lambda)$ the mean patch size is calculated as the average of the value $\left\langle B_{s}\right\rangle_{T_{1}<t<T_{f}}$ over 30 runs. Clearly a global maximum of the patch size is obtained for intermediate values of $\tau=\tau_{c} \approx 9-17$ days and $\lambda=\lambda_{c} \approx 140-250 \mathrm{~km}$.

In order to study the influence of the mixing properties of the chosen flow on the phytoplankton growth, we measure the mixing efficiency $\varsigma(\tau, \lambda, t)$ in terms of finite-time Lyapunov exponents $\Lambda(\boldsymbol{r}, t)$ (FTLE, see Appendix A). For a certain Gaussian flow with a constant time scale $\tau$ and length scale $\lambda$, the mean mixing efficiency is defined as the mean of the FTLE values over the $3 \times 3$ area of the nutrient source

$\varsigma(\tau, \lambda, t)=\langle\Lambda(\boldsymbol{r}, t)\rangle_{3 \times 3}$,

to quantify the relevant mixing for the plankton patch. Fig. 5 shows the mean mixing efficiency $\varsigma$ ( $t=30$ days) as a function of the parameters $(\tau, \lambda)$. The parameter space is the same as in Fig. 4. Note that the maximum value of the mixing efficiency $\varsigma(\tau, \lambda)$ is obtained for different values of $(\tau, \lambda)$ as the 


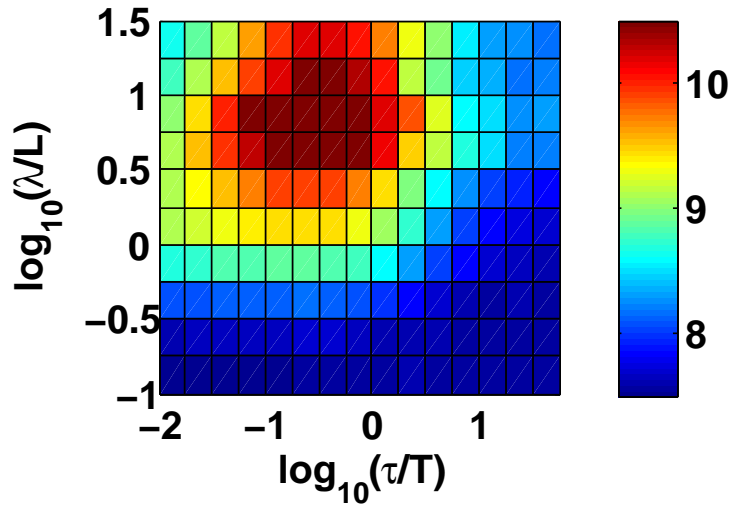

Fig. 4. Mean phytoplankton patch size $\left\langle B_{S}\right\rangle \times 10^{-5} \mathrm{~km}^{2}$ as a function of time and length scales $(\tau, \lambda)$ of the Gaussian flow, Eqs. (24). Optimal Eulerian time and length scales are approximately $\left(\log _{10} \tau_{c}, \log _{10} \lambda_{c}\right)=(-0.5,0.875)$. Each data point shown in the figure has been calculated as an average over 30 different initial conditions for the flow. Set of parameters as in Fig. 3.

maximum patch size in Fig. 4. A flow with a maximum mixing efficiency provides optimal mixing for passive tracers, whereas a comparison between Figs. 4 and 5 suggests that reactive plankton does not spread optimally under the same conditions.

The mixing efficiency calculated from FTLE values can also be interpreted as a Lagrangian time scale $1 / 5$ of the flow, representing the typical time for tracer separation along the trajectory of the patch. It is obvious from Fig. 5 that, for the Gaussian flow, this Lagrangian time scale depends on both the Eulerian time and length scale. For optimal Eulerian values $\left(\tau_{c}, \lambda_{c}\right)$ concerning patch size, the Lagrangian time scale is $\tau_{L}=1 / \varsigma\left(\tau_{c}, \lambda_{c}\right) \approx 10$ days which is approximately the typical reaction time $T_{1} \approx 9$ days the plankton needs to grow after the switch-on of the nutrient source. Faster mixing seems unfavourable for the spread of the plankton patch, because the patch is diluted before having grown: slower mixing reduces the patch size due to a lack of transport. Although the optimal Lagrangian time scale is provided by the flow for a range of $(\tau, \lambda)$ values (yellow circle in Fig. 5), the maximal patch size is only obtained for large Eulerian length scales of the flow.

From the previous academic flow model, we found that there exists optimal time and length scales favouring phytoplankton growth for the used NPZ model. For these optimal scales, the front becomes much more distorted than for the other scales and the final patch area is the largest. Observations of the dynamics of the modelled phytoplankton patch show that flow regions of low velocity values (approximate constant stream function) trap and confine the plankton in "optimal conditions" while the bloom occurs. The surrounding filaments with larger velocity values tend to transport the plankton while not allowing it to grow laterally towards the transport direction. This indicates that optimal time and length scales are needed to promote these optimal conditions.

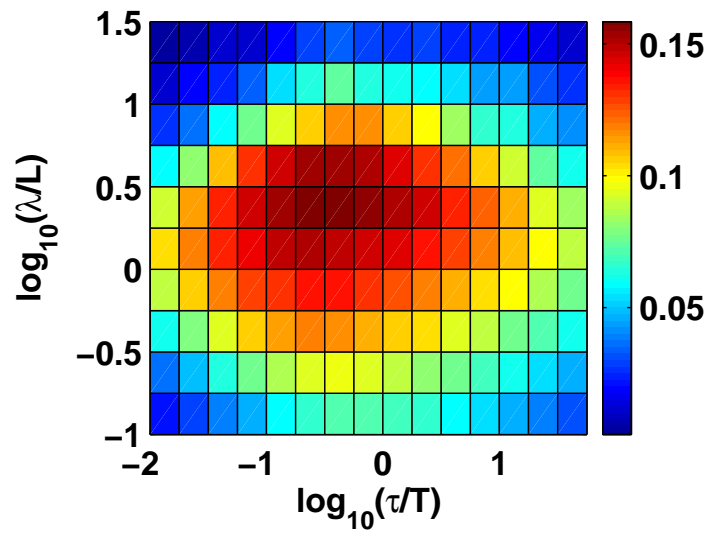

Fig. 5. Mean mixing efficiency $\varsigma(\tau, \lambda)\left[\right.$ days $\left.^{-1}\right]$ in terms of FTLE values at the nutrient source Eq. (11) as a function of Eulerian time and length scales $(\tau, \lambda)$ for the Gaussian flow, Eqs. (2-4). Set of parameters as in Fig. 3.

\subsection{Altimetry flow}

In this section, we analyse the development of phytoplankton patches for a more realistic flow model derived from satellite altimetry data, Eq. (5). We chose a multiscale region of the North Atlantic, where eddies with different length scales are clearly visible (Fig. 1b). Although the real altimetry flow changes over the integration time of 30 days, we use a simplified stationary flow. This allows for a better local definition of time and length scales and reveals well-defined flow structures that influence the plankton spreading. As in the Gaussian flow, a $3 \times 3$ constant nutrient source is set with its centre positioned at each grid point of the $130 \times 90$ domain to initiate a plankton patch. Then the phytoplankton patch evolving from each of these nutrient sources is modelled with the NPZ model. After 30 days the final patch size $B_{\mathrm{S}}$ is calculated as in Eq. (10). Figure 6 shows the patch size as a function of the position of the nutrient source in the altimetry flow. It is obvious that the position of the nutrient source in the flow strongly affects the evolution and, therefore, the final size of the plankton bloom.

In order to compare the phytoplankton growth in the altimetry flow to that in the Gaussian flow, we compute time and length scales for each point in the constant altimetry velocity field. As Lagrangian time scale $\tau_{L}$ we use again the local reciprocal Finite-Time Lyapunov Exponent $\tau_{L}=1 / \Lambda$ at the nutrient source. The local length scale $\lambda(\boldsymbol{x})$ for the altimetry flow is derived from the autocorrelation function $\Phi(\boldsymbol{x}, r, \lambda)$ of the velocity field assuming an exponential decay,

$$
\begin{aligned}
\phi(\boldsymbol{x}, r) & =\left\langle\frac{\boldsymbol{v}(\boldsymbol{x}) \boldsymbol{v}(\boldsymbol{x}+\boldsymbol{r})}{|\boldsymbol{v}(\boldsymbol{x})||\boldsymbol{v}(\boldsymbol{x}+\boldsymbol{r})|}\right\rangle_{r=|\boldsymbol{r}|=\mathrm{const}} \\
& =\exp \left(-\frac{r}{\lambda(\boldsymbol{x})}\right) .
\end{aligned}
$$




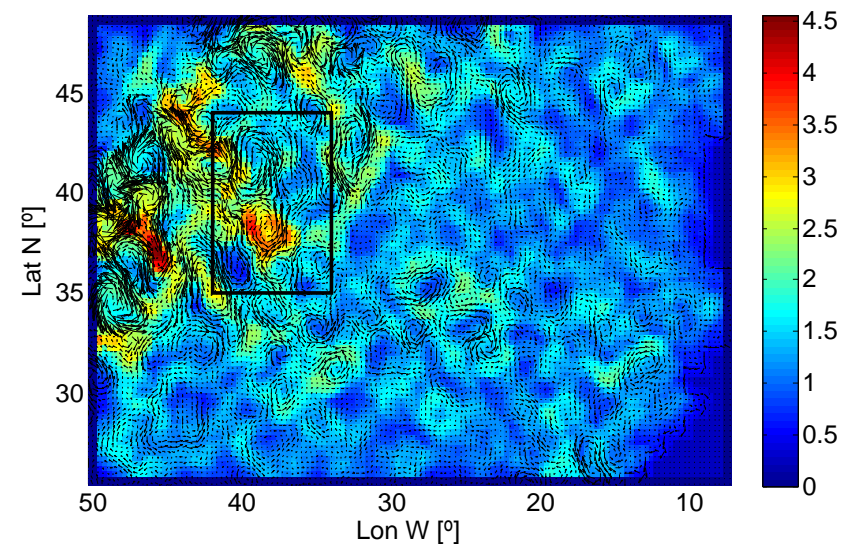

Fig. 6. Phytoplankton patch size $B_{\mathrm{S}} \times 10^{-5} \mathrm{~km}^{2}$ after 30 days depending on the position of the nutrient source in the stationary altimetry flow. Especially in the black box (also marked in Fig. 1b) strong differences in patch size are obvious.

Thus, we obtain an Eulerian length scale for the altimetry flow that is very similar to the a priori Eulerian length scale of the Gaussian flow model, which is also an exponentially decaying autocorrelation length. The patch size data for both flow models is binned to these time and length scales, whereby we discarded the data with few configurations for $\left(\tau_{L}, \lambda\right)$ values and, therefore, high errors. Figure 7 shows the phytoplankton patch size as a function of Lagrangian time and Eulerian length scales for the Gaussian model flow (panel a) and the altimetry flow (panel b). For both a clear maximum cannot be observed, as not the whole parameter space can be covered, but both show a very similar dependence of the patch size on time and length scales. Figure 7 suggests that, for the covered parameters, both flows provide optimal conditions for plankton spread at length scales $\lambda_{c} \approx 140-250 \mathrm{~km}$ and $\tau_{c} \approx 5-15$ days.

Apart from the statistical analysis concerning time and length scales, it is worthwhile to have a closer look at a selected region of the flow, to analyse the interplay of the different parameters on the plankton spreading. The selected region is marked in the stream function image (Fig. 1) and in the patch size image (Fig. 6). Note that, in the boxed area, the overall patch size in the lower eddy is significantly larger than in the upper one, although both eddies have similar velocities and diameters.

Figure 8 shows a close-up of the region with two eddies, marked with letters A and B. In order to understand the different dynamics in both eddies that lead to the variation in plankton patch size, we exemplary chose two points, one in each eddy, with a strong difference in patch size. Both sources are positioned in the rotating eddy with a similar distance to the centre, respectively. Figure 8a, b shows the two phytoplankton distributions that developed from the chosen points after 30 days. For graph a the patch size is clearly larger than for graph b. Figure 9 helps to understand these differences. It shows the plankton patch size in the region of
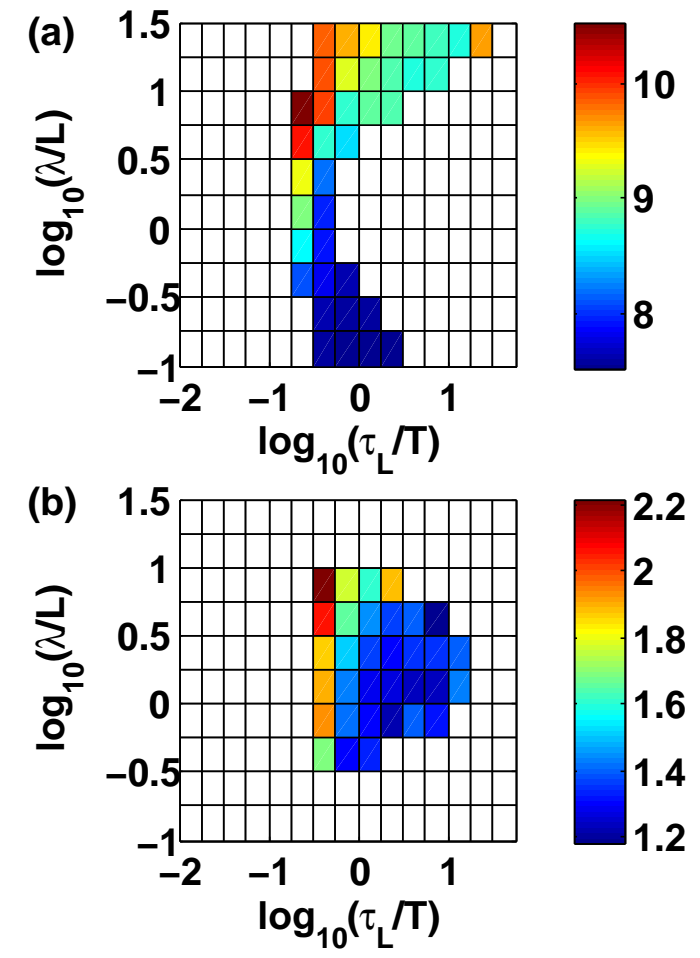

Fig. 7. Phytoplankton patch size $B_{\mathrm{S}} \times 10^{-5} \mathrm{~km}^{2}$ as a function of Eulerian length scale (Eq. 12) and Lagrangian time scale $\tau_{L}=1 / \Lambda$ for the Gaussian flow (a) and the altimetry flow (b).

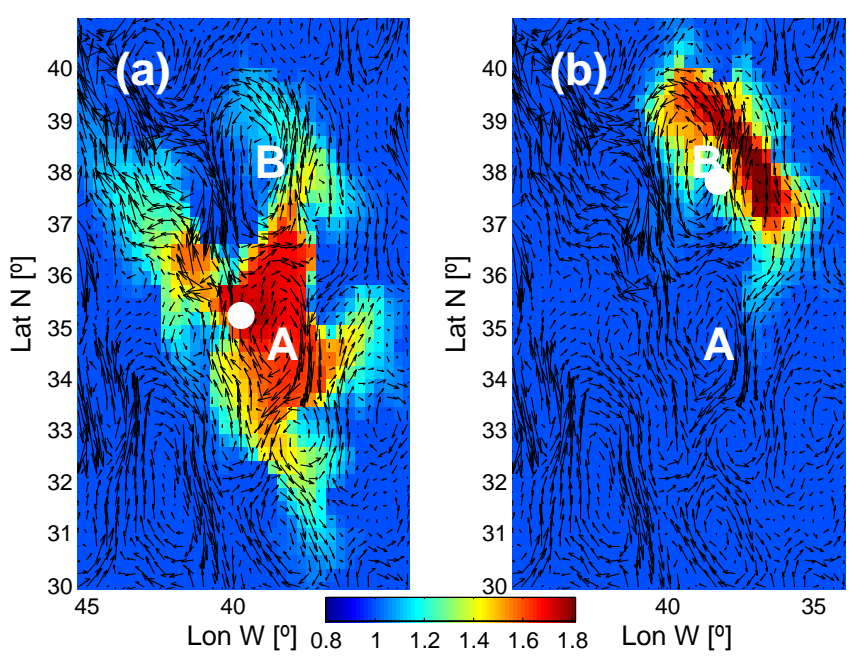

Fig. 8. Phytoplankton concentration $P / P_{0}$ for two different patches in the North Atlantic ocean after 30 days of simulation initiated at the white dot, respectively. Letters A and B mark the centres of eddies of similar size.

interest (panel a), the local Eulerian length scales (panel b), Lagrangian time scales (panel c) and the flow structure with hyperbolic points (panel d) obtained from an analysis of Finite-Time Lyapunov Exponents. The plankton patch in eddy $\mathrm{A}$ is advected around the eddy in optimal time and 
(a) Phytoplankton patch size $\left[10^{5} \mathrm{~km}^{2}\right]$
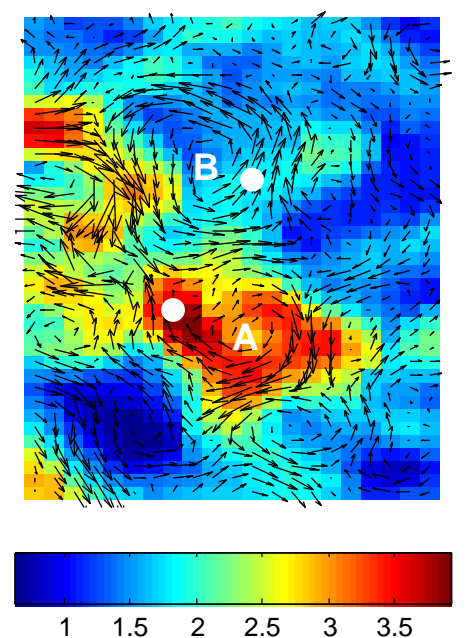

(c) Time scale $1 / \Lambda$ [days]

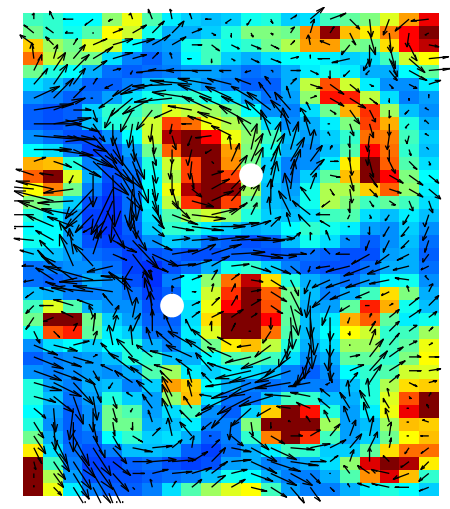

(b) Local length scale $[\mathrm{km}]$
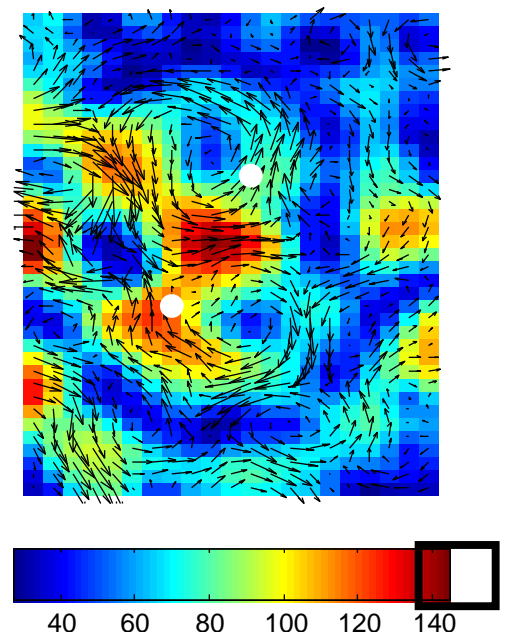

(d) $\Lambda^{+}-\Lambda^{-}[1 /$ days $]$
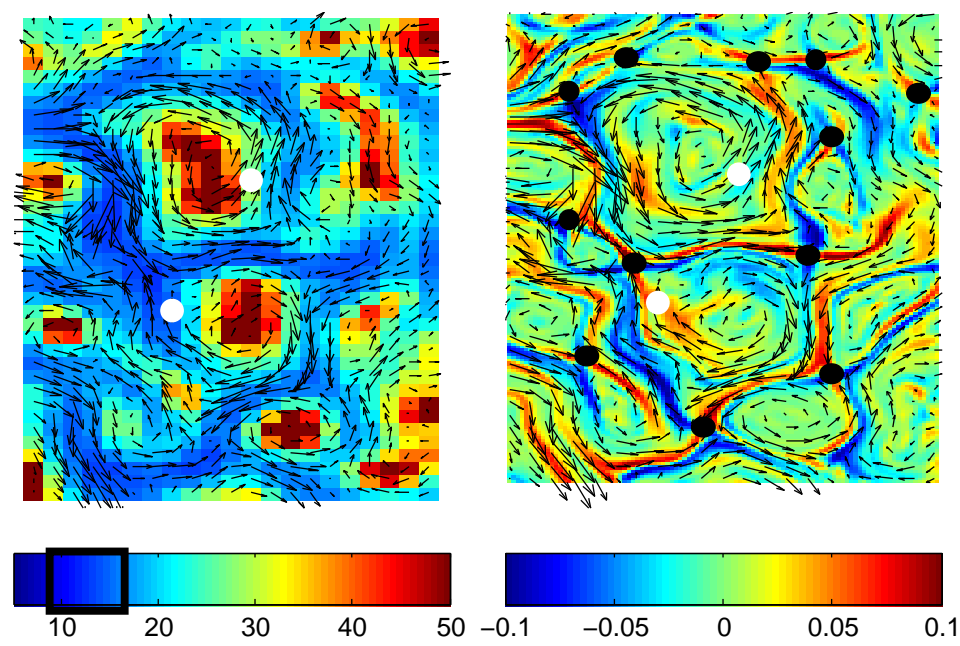

Fig. 9. Discrepancy in plankton growth in two apparently similar eddies: Size of plankton patch for a selected region in the North Atlantic Ocean after 30 days of simulation (a). For each grid point, colour indicates the patch size $B_{\mathrm{S}} \times 10^{-5} \mathrm{~km}^{2}$ after a nutrient upwelling took place at that location. Red/blue colour shows areas with larger/smaller phytoplankton patch. In eddy A, plankton growth is favoured. The representations of Eulerian length scales and Lagrangian time of the flow (b, c) show that both long length scales $(\lambda>120 \mathrm{~km})$ and short time scales $\left(\tau_{L}<15\right.$ days) are necessary for a large plankton patch size. Black boxes at the colour bars show the optimal time and length scales found for the Gaussian correlated flow. Finite-Time Lyapunov Exponents (d) for the same region and period of time as for panels (a, b, c) calculated forward (displayed as positive values) and backward (displayed as negative values) integrations in time. The plotted field is $\Lambda^{+}-\left|\Lambda^{-}\right|$(see Appendix A). Mesoscale structures with jets and vortices can clearly be observed. The black dots indicate the hyperbolic points that are located at the intersections of the stable (red) and unstable (blue) manifolds. Eddy A is surrounded by 5 hyperbolic points with the same distance. They act as exits to other favourable regions. The hyperbolic points surrounding eddy B have smaller and less uniform distances. White dots correspond to the positions of the exemplary nutrient sources in Fig. 8

length scales while it successively passes three hyperbolic points that spread the plankton to neighbouring regions with favourable conditions too. It, therefore, forms four arms. In contrast to this, the patch initiated in eddy B is, indeed, also spread by one hyperbolic point, but into a western region of small length scales and into an eastern region with long time scales, so the patch development is hindered. As a result, not only does the local time and length scales of the flow influence the growth of the plankton patch, but also the connection between regions with favourable conditions for plankton growth, which can only be seen in maps of Lagrangian quantities like the FTLE. Stable and unstable manifolds in the flow govern the transport of tracers (Lehahn et al., 2007), while hyperbolic points mark the positions where these manifolds cross and the direction of the velocity changes abruptly. 


\section{Conclusions}

The effect of time and length scales of two different fluid flows on phytoplankton growth has been analysed.

In both cases, we found that growth is enhanced for a critical time scale comparable to the one associated with the biological system, as it was also pointed out in previous studies (Medvinsky et al., 2001; Sandulescu et al., 2007, 2008). We found an optimal Eulerian time scale for the Gaussian flow of $\tau_{c} \approx 9-17$ days and optimal Lagrangian time scales of $\tau_{c} \approx 5-15$ days for the Gaussian and the altimetry flow, where the relevant time scale for the advected plankton system is the Lagrangian one. We observed that for a critical Eulerian length scale of the order of $140-250 \mathrm{~km}$, the patch size attains a maximum for the Gaussian flow. For this length scale, the front becomes more distorted than for other scales and the final patch area is the largest. A similar result is found for the altimetry flow.

In terms of spatial Lagrangian properties as the FTLE field or stable/unstable manifolds, a certain length scale is needed to balance two unfavourable conditions: on the one hand, for shorter length scales the flow rapidly mixes the plankton and does not allow long scale transport, and on the other hand, for longer length scales few hyperbolic points are present in the medium and due to the lack of separatrices (stable and unstable manifolds) the flow does not favour the spreading of the initial patch in different directions. Thus, length scales in the FTLE field of a flow can indicate regions where a large plankton bloom size is expected. However, caution must be urged with this approach as the present study does not take into account many physical and biological processes that affect plankton growth such as vertical upwelling, sea temperature, or the depth of the mixed layer and does not resolve explicitly 3-D effects.

\section{Appendix A}

\section{FTLE calculations}

The importance of Lagrangian analysis to understand ocean dynamics has been established during the last decade (see Griffa et al. (2007) and Neufeld and Hernández-García (2009) and references therein for a review). Among other techniques, Finite-Time Lyapunov Exponents (FTLE) are used to quantify mixing and patterns in the spatial FTLE field reveal coherent structures in the flow. FTLE values are computed from the trajectories of Lagrangian tracers in the flow. Therefore, a regular grid of tracers is advected by the velocity field with a linear integration scheme and a bicubic interpolation of the velocity field to the tracer positions. After the finite time $\tau$, the FTLE fields are computed from the final tracer positions (Mancho et al., 2006)

$\Lambda(\mathbf{x})=\frac{1}{\tau} \ln \sqrt{\lambda_{\max }(\Delta(\mathbf{x}))}$, where $\lambda_{\max }$ is the largest eigenvalue of the finite-time Cauchy-Green deformation tensor $\Delta$, calculated from the flow map of the tracers. $\lambda_{\max }$ denotes the ratio of stretching between two initially close tracers in the direction of the largest stretching. The finite time $\tau$ is chosen to have the same duration as the plankton simulation, i.e. $\tau=30$ days, to detect relevant Lagrangian structures for the plankton spreading. The initial distance of the tracers is $37 \mathrm{~km}$ to obtain a Lagrangian time scale (Fig. 9c), which is the same grid as the plankton simulation. A finer grid of $7.4 \mathrm{~km}$ is used to obtain FTLE ridges and hyperbolic points (Fig. 9d). Fig. 9d shows the difference of FTLE fields $\Lambda^{ \pm}(-)$calculated forward $\left(\Lambda^{+}\right)$and backward $\left(\Lambda^{-}\right)$advection of the tracers in time (d'Ovidio et al., 2004, 2009),

$\Lambda^{ \pm}(-)=\Lambda^{+}-\left|\Lambda^{-}\right|$

Unstable manifolds, with a flow towards the hyperbolic point, appear with positive values and stable manifolds, away from the hyperbolic point, with negative values. Hyperbolic points can be extracted by determining the local maxima in the added field $\Lambda^{ \pm}(+)$(not shown here).

Acknowledgements. This work was financially supported by Xunta de Galicia through the project DRIFTER (ERACCT2005016165) within the framework of the EU ERA-Net initiative (6th Framework Program) and the Research Grant No. PGIDIT07PXIB206077PR. We thank E. Hernández-García for helpful discussions.

Edited by: S. Wiggins

Reviewed by: two anonymous referees

\section{References}

Abraham, E. R.: The generation of plankton patchiness by turbulent stirring, Nature, 391, 577-580, 1998.

Alonso, S., Sagués, F., and Sancho, J. M.: Excitability transitions and wave dynamics under spatiotemporal structured noise, Phys. Rev. E, 65, 066107(1)-066107(11), 2002.

Bainbridge, R.: The size, shape and density of marine phytoplankton concentrations, Biol. Rev., 32, 91-115, 1957.

Beltrami, E. and Carroll, T. O.: Modeling the role of viral disease in recurrent phytoplankton blooms, J. Math Biol., 32, 857-863, 1994.

Bracco, A., Clayton, S., and Pasquero, C.: Horizontal advection, diffusion, and plankton spectra at the sea surface, J. Geophys. Res., 114, C02001, doi:10.1029/2007JC004671, 2009.

Fasham, M., Ducklow, H., and McKelvie, S.: A nitrogen-based model of plankton dynamics in the oceanic mixed layer, J. Mar. Res., 48, 591-639, 1990.

Franks, P. J. S.: NPZ models of plankton dynamics: Their construction, coupling to physics, and application, J. Oceanogr., 58, 379-387, 2002.

Griffa, A., Kirwan, A. D., Mariano, A. J., Ozgokmen, T., and Rossby, T. (Eds.): Lagrangian analysis and prediction of coastal and ocean dynamics, Cambridge Univ. Press, 2007. 
Griffiths, B. M.: Early references to water blooms in British Lakes, Proceedings of the Linnean Society of London, 151, 12-19, 1939.

Haller, G. and Yuan, G.: Lagrangian coherent structures and mixing in two-dimensional turbulence, Physica D, 147, 352-370, 2000.

Lehahn, Y., d'Ovidio, F., Lévy, M., and Heifetz, E.: Stirring of the northeast Atlantic spring bloom: A Lagrangian analysis based on multisatellite data, J. Geophys. Res., 112, C08005, doi:10.1029/2006JC003927, 2007.

Lévy, M. and Klein, P.: Does the low frequency variability of mesoscale dynamics explain a part of the phytoplankton and zooplankton spectral variability?, Proc. R. Soc. Lon. A, 460, 16371687, 2004.

Lévy, M.: The Modulation of Biological Production by Oceanic Mesoscale Turbulence, Lect. Notes Phys., 744, 219-261, 2008.

Lorenzo, M. N., Santos M. A., and Pérez-Muñuzuri, V.: Spatiotemporal stochastic forcing effects in an ensemble consisting of arrays of diffusively coupled Lorenz cells, Chaos, 13, 913-920, 2003.

Mahadevan, A. and Archer, D.: Modeling the impact of fronts and mesoscale circulations on the nutrient supply and biogeochemistry of the upper ocean, J. Geophys. Res., 105(C1), 1209-1221, 2000.

Mahadevan, A. and Tandon, A.: Ananalysis of mechanisms for submesoscale vertical motion at ocean fronts, Ocean Model., 14, 241-256, 2006.

Mancho, A. M., Small, D., and Wiggins, S.: A tutorial on dynamical systems concepts applied to Lagrangian transport in oceanic flows defined as finite time data sets; Theoretical and computational issues, Phys. Rep.., 437, 55-124, 2006.

Martin, A. P.: On filament width in oceanic plankton distributions, J. Plankton Res., 22, 597-602, 2000.

Martin, A. P., Richards, K. J., and Fasham, M. J.: Phytoplankton production and community structure in an unstable frontal region, J. Marine Syst., 28, 65-89, 2001.

Martin, A. P., Richards, K. J., Bracco, A., and Provenzale, A.: Patchy productivity in the open ocean, Global Biogeochem. Cy., 16, 1025-1033, 2002.

Martin, A. P.: Phytoplankton patchiness: the role of lateral stirring and mixing, Prog. Oceanogr., 57, 125-174, 2003.

McKiver, W., Neufeld, Z., and Scheuring, I.: Plankton bloom controlled by horizontal stirring, Nonlin. Processes Geophys., 16, 623-630, 2009, http://www.nonlin-processes-geophys.net/16/623/2009/.

McLeod, P., Martin, A. P., and Richards, K. J.: Minimum length scale for growth -limited oceanic plankton distributions, Ecol. Model., 158, 111-120, 2002.
Medvinsky, A. S., Tikhonova, I. A., Rubin, R. A., Li, B. L., Lin, Z. S., and Malchow, H.: Patchy environment as a factor of complex plankton dynamics, Phys. Rev. E., 64, 021915, doi:10.1103/PhysRevE.64.021915, 2001.

Neufeld, Z. and Hernández-García, E.: Chemical and biological processes in fluid flows, Imperial College Press, 2009.

Okubo, A.: Oceanic diffusion diagrams, Deep-Sea Res., 18, 789$802,1971$.

Oschlies, A. and Garcon, V.: Eddy-induced enhancement of primary production in a model of the North Atlantic Ocean, Nature, 394, 266-269, 1998.

Oschlies, A. and Garcon, V.: An eddy-permitting coupled physicalbiological models of the North Atlantic: 1 . Sensitivity to advection numerics and mixed layer physics, Global Biogeochem. Cy., 13, 135-160, 1999.

d'Ovidio, F., Fernández, V., Hernández-García, E., and López, C.: Mixing structures in the Mediterranean Sea from finite-size Lyapunov exponents, Geophys. Res. Lett., 31, 17203-17206, 2004.

d'Ovidio, F., Isern-Fontanet, J., López, C., Hernández-García, E., and García-Ladona, E.: Comparison between Eulerian diagnostics and finite-size Lyapunov exponents computed from altimetry in the Algerian basin, Deep-Sea Res. Pt. I, 56, 15-31, 2009.

Pasquero, C., Bracco, A., and Provenzale, A.: Impact of the spatiotemporal variability of the nutrient flux on the primary productivity in the ocean, J. Geophys. Res., 110, C07005, doi:10.1029/2004JC002738, 2005.

Sagués, F., Sancho, J. M., and García-Ojalvo, J.: Spatiotemporal order out of noise, Rev. Mod. Phys., 79, 829-880, 2007.

Sandulescu, M., López, C., Hernández-García, E., and Feudel, U.: Plankton blooms in vortices: the role of biological and hydrodynamic timescales, Nonlin. Processes Geophys., 14, 443-454, 2007,

http://www.nonlin-processes-geophys.net/14/443/2007/.

Sandulescu, M., López, C., Her4nández-García, E., and Feudel, U.: Biological activity in the wake of an island to a coastal upwelling, Ecol. Complex., 5, 228-237, 2008.

Spiegelman, M. and Katz, R. F.: A semi-Lagrangian CrankNicolson algorithm for the numerical solution of advectiondiffusion problems, Geochem. Geophy. Geosy., 7, Q04014, doi:10.1029/2005GC001073, 2006.

Staniforth, A. and Cote, J.: Semi-Lagrangian integration schemes for atmospheric models - a review, Mon. Weather Rev., 119, 2206-2223, 1991.

Wiggins, S.: The dynamical systems approach to Lagrangian transport in oceanic flows, Annu. Rev. Fluid Mech., 37, 295-328, 2005. 